OPEN ACCESS

Edited by:

Valerie Purvin

Midwest Eye Institute, United States

Reviewed by:

Gianluca Coppola,

Sapienza University of Rome, Italy

Simona Maccora,

University of Palermo, Italy

*Correspondence:

Ozan E. Eren

ozan.eren@med.uni-muenchen.de

tThese authors have contributed equally to this work

Specialty section:

This article was submitted to

Neuro-Ophthalmology,

a section of the journal

Frontiers in Neurology

Received: 26 January 2021 Accepted: 15 March 2021

Published: 04 May 2021

Citation:

Eren OE, Ruscheweyh R, Rauschel V, Eggert T, Schankin CJ and Straube A

(2021) Magnetic Suppression of

Perceptual Accuracy Is Not Reduced in Visual Snow Syndrome.

Front. Neurol. 12:658857.

doi: 10.3389/fneur.2021.658857

\section{Magnetic Suppression of Perceptual Accuracy Is Not Reduced in Visual Snow Syndrome}

\author{
Ozan E. Eren ${ }^{1 *}$, Ruth Ruscheweyh ${ }^{1}$, Veronika Rauschel ${ }^{1}$, Thomas Eggert ${ }^{1}$, \\ Christoph J. Schankin ${ }^{1,2 \dagger}$ and Andreas Straube ${ }^{1 \dagger}$ \\ ${ }^{1}$ Department of Neurology, Ludwig Maximilians University of Munich, University Hospital - Großhadern, Munich, Germany, \\ ${ }^{2}$ Department of Neurology, Inselspital, Bern University Hospital, University of Bern, Bern, Switzerland
}

Objective: Patients with visual snow syndrome (VSS) suffer from continuous ("TV snow-like") visual disturbance of unknown pathoetiology. In VSS, changes in cortical excitability in the primary visual cortex and the visual association cortex are discussed, with recent imaging studies tending to point to higher-order visual areas. Migraine, especially migraine with aura, is a common comorbidity. In chronic migraine and episodic migraine with aura but not in episodic migraine without aura, a reduced magnetic suppression of perceptual accuracy (MSPA) reflects a probably reduced inhibition of the primary visual cortex. Here we investigated the inhibition of the primary visual cortex using MSPA in patients with VSS, comparing that with MSPA in controls matched for episodic migraine.

Methods: Seventeen patients with VSS were compared to 17 age- and migraine-matched controls. Visual accuracy was assessed by letter recognition and modulated by transcranial magnetic stimulation delivered to the occipital cortex at different intervals with respect to the letter presentation (40, 100, and $190 \mathrm{~ms}$ ).

Results: Suppression of visual accuracy at the 100-ms interval was present without significant differences between VSS patients and age- and migraine-matched controls (percentage of correctly recognized trigrams, control: $46.4 \pm 34$.3; VSS: $52.5 \pm 25.4$, $p=0.56)$.

Conclusions: In contrast to migraine with aura, occipital cortex inhibition, as assessed with MSPA, may not be affected in VSS.

Keywords: visual snow syndrome, cortical hyperexcitability, magnetic suppression of perceptual accuracy, migraine, pathophysiology

\section{INTRODUCTION}

Patients with visual snow (VS) describe continuous, mostly black and white tiny flickering dots in their entire visual field, comparable to the old TV-static noise when missing the analog signal. When accompanied by other visual symptoms such as afterimages (palinopsia), impaired night vision (nyctalopia), or increased light sensitivity (photophobia), it is called visual snow syndrome (VSS) (1-3). Its pathophysiology is still under discussion, and although it is highly associated with migraine with and without aura and may partially overlap with these, recent research strongly suggests that VS is a distinct disorder (2-5). The visual disturbance sums up to a clinical picture that 
is best explained by dysfunction of the higher-order visual cortex. Consistently, fluorodeoxyglucose-positron emission tomography (PET) investigations showed hypermetabolism in the lingual gyrus, an area of the higher visual association cortex $(3,6)$. Importantly, these findings from functional neuroimaging were confirmed by voxel-based morphometry by two independent groups which demonstrated increased gray matter volume in the same cortical area $(6,7)$. A possible neurophysiological correlate of the involvement of higher visual areas could be the significantly prolonged latency of the late N145 potentials with normal P100 potentials in visual evoked potentials (4). However, the picture is likely more complex with studies pointing to a dysfunction of the primary visual cortex, considering thalamocortical dysrhythmia as the origin of VSS $(8,9)$ and demonstrating alterations also in non-visual, acoustic, and limbic areas (6).

Here we used magnetic suppression of perceptual accuracy (MSPA) to further elucidate the role of the primary visual cortex or at least its inhibition in VSS. Reduced MSPA reflects reduced inhibition of the primary visual cortex, which is seen in chronic migraine and episodic migraine with aura, but not in episodic migraine without aura (10). Reduced MSPA in VSS would therefore argue for a decreased local inhibition of the primary visual cortex similar to episodic migraine with aura.

\section{MATERIALS AND METHODS}

The study was conducted in accordance with the Declaration of Helsinki and approved by the local ethics committee (227-15). All patients gave written informed consent. The results of the study were presented in preliminary format at the International Headache Conference 2017 (11).

\section{Subjects}

For recruitment, the study was advertised in social media with support from the self-help group on VS, "Eye on Vision Foundation" (http://www.eyeonvision.org/). We first assessed the eligibility of interested patients during telephone interviews conducted by a headache specialist familiar with VSS. The interview was cross-checked by a second headache specialist. Inclusion criteria were age $\geq 18$ years and presence of VSS (subtype black and white dots) in accordance with the criteria published previously (2). Exclusion criterion was intake of any illicit drugs currently or within 2 weeks prior to the onset of VSS. Brain MRI was normal in all subjects. Later, the VSS patients were examined at presentation by one of the two mentioned specialists. Of medications known to possibly affect cortical excitability, only one patient was on lamotrigine and three patients were on mirtazapine. Travel expenses were reimbursed, and no further payment was made for study participation. Patients with VSS were compared to age- and migraine-matched subjects.

\section{Measurement of MSPA}

MSPA was measured according to our previous work (10). To summarize, three-letter sequences (so-called trigrams) were presented for $30 \mathrm{~ms}$ on a monitor in front of the subjects. They were instructed to read the letters aloud from left to right. In a first step, training runs were performed to adjust contrast in a manner that $\sim 80 \%$ of the letters could be recognized correctly by the subject without transcranial magnetic stimulation (TMS) intervention. In a second step, the experiment was started by presenting a series of 54 trigrams, followed each by a TMS pulse (output of at least $70 \%$ of the possible maximum output, Magstim 200, The MagStim Company Ltd, Whitland, UK) via a $90-\mathrm{mm}$ circular coil to the occipital cortex in randomized intervals of 40,100 , or $190 \mathrm{~ms}$ in regard to the trigram presentation. The time between the start of trigram presentation and TMS pulse delivery is called stimulus onset asynchrony (SOA). Later, the percentage of correctly recognized trigrams was calculated for each SOA interval. All subjects were measured interictally; as corroborated by telephone contact, no subject reported a migraine attack or aura within 2 days after the experiment.

\section{Statistical Analysis}

Statistical analysis was done using SPSS 25 (IBM Corp. Released 2017. IBM SPSS Statistics for Windows, version 25.0.0.1, 32-bitversion, Armonk, NY: IBM Corp.). Statistical significance was assumed at $p \leq 0.05$. The demographics of the groups were compared using chi-square test.

ANOVA was used for MSPA comparison (within-subject factor: SOA, between-subject factor: group). Where ANOVA was significant $(p \leq 0.05), t$-test with Bonferroni correction was used for post hoc analysis.

\section{Data Availability Statement}

Anonymized data will be shared at request from any qualified investigator.

\section{RESULTS}

\section{Subjects}

Seventeen patients with visual snow syndrome (six females and 11 males; mean age, $30.0 \pm 10.8$ years; 12 with comorbid migraine, seven of them also with typical migraine aura) were compared to 17 control subjects (C) (14 females and three males; mean age, $28.3 \pm 8.2$ years; 12 with comorbid migraine, none of them with typical migraine aura). The groups did differ in gender $(\chi=7.77 p=0.005)$ and aura $(\chi=8.82 p=$ $0.003)$, but not in migraine $(\chi=0, p=1)$ and age $\chi=$ $16.87, p=0.66$. For more information on study population, see the Supplementary Table 1. If not stated otherwise, the term "controls" describes the group of age- and migrainematched subjects.

\section{MSPA}

The MSPA profiles of patients with VSS and migraine-matched controls can be seen in Figure 1 and Table 1.

There was a significant main effect of SOA interval (rmANOVA: Greenhouse-Geisser $F$ 31.75, $p \leq 0.01$ ) with a significant suppression of visual accuracy at $100 \mathrm{~ms}$ compared to 40 and $190 \mathrm{~ms}$. There was no significant main effect of group (rmANOVA: $F$ 0.70, $p=0.47$ ). Additionally, we 


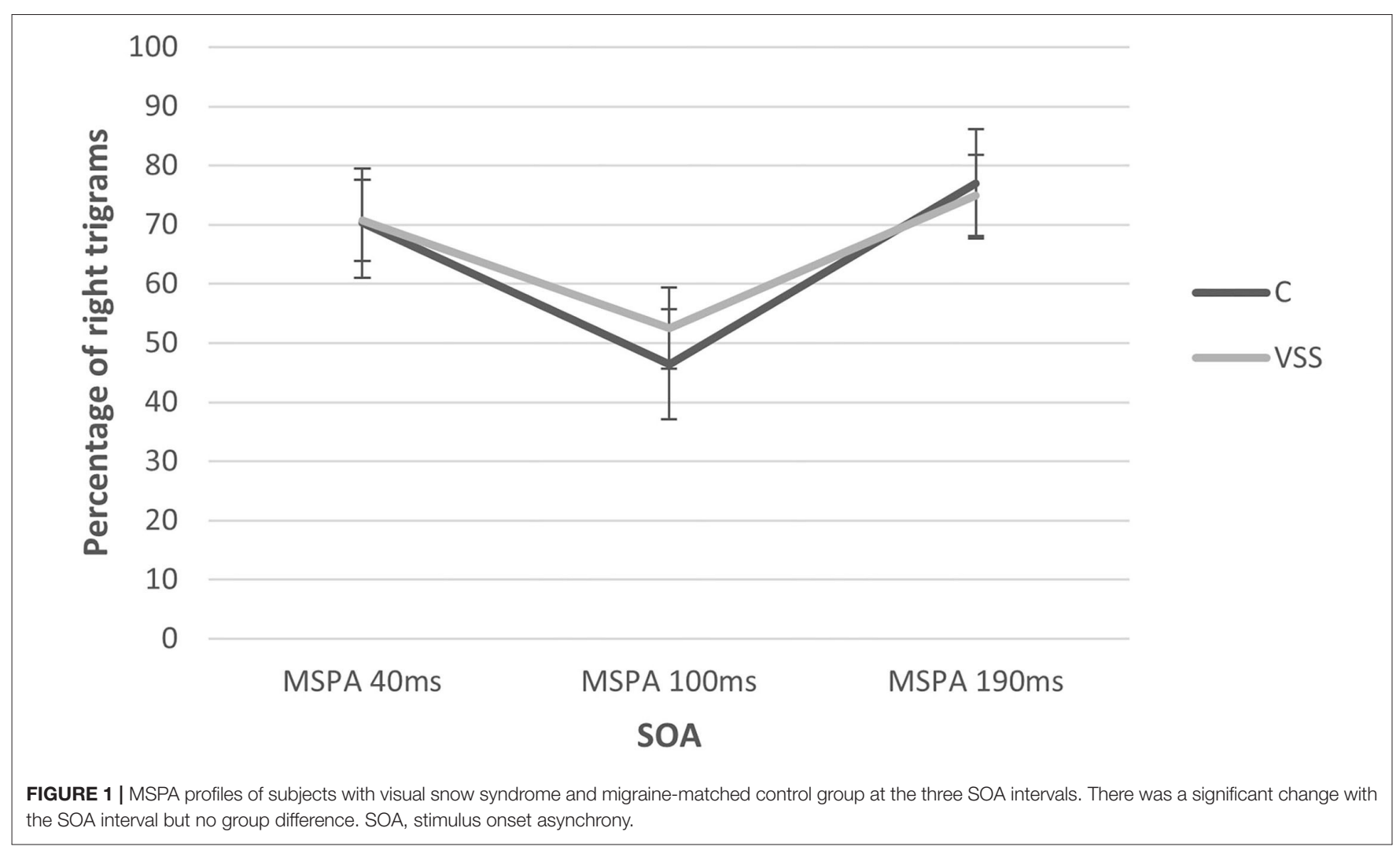

TABLE 1 | Mean percentage of correctly detected trigrams at the three different stimulus onset asynchrony (SOA) intervals (40, 100 and $190 \mathrm{~ms}$ ).

\begin{tabular}{lcc}
\hline SOA & \multicolumn{2}{c}{$\begin{array}{c}\text { Average percentage correct } \\
\text { (mean } \pm \text { SD) }\end{array}$} \\
\cline { 2 - 3 } & Visual snow syndrome & Control \\
\hline $40 \mathrm{~ms}$ & $70.70 \pm 14.88$ & $70.26 \pm 26.53$ \\
$100 \mathrm{~ms}$ & $52.51 \pm 25.41$ & $46.40 \pm 34.26$ \\
$190 \mathrm{~ms}$ & $74.95 \pm 10.08$ & $76.91 \pm 22.62$ \\
\hline
\end{tabular}

performed an explorative subgroup analysis beginning with comparing the same control group only with VSS patients without migraine aura, showing almost identical percentages of correctly recognized trigrams at $100 \mathrm{~ms}$ (C: $46.40 \pm 34.26$ vs. VSS: $46.67 \pm 27.99)$, again without significant group differences (rmANOVA: $F$ 0.261, $p=0.77$ ).

Afterwards, to understand the effect of aura in our VSS group itself, we compared VSS patients with migraine with aura (VSSMwA; $n=7$ ) and VSS patients with migraine without aura (VSSMwoA; $n=5$ ). There was again a significant main effect of SOA interval (rmANOVA: Greenhouse-Geisser F 6.31, $p \leq$ $0.05)$ with a significant suppression of visual accuracy at $100 \mathrm{~ms}$ (VSSMwoA: $48.2 \pm 31.89$ vs. VSSMwA: $60.85 \pm 20.21$ ) compared to 40 and $190 \mathrm{~ms}$, but also no significant main effect of group (rmANOVA: $F$ 0.40, $p=0.57$ ).

\section{DISCUSSION}

The main result of this study is that magnetic suppression of perceptual accuracy is not reduced in visual snow syndrome when compared to controls matched for migraine. In this respect, VSS differs from migraine with aura (12). The significant suppression at $100 \mathrm{~ms}$ is comparable instead to that of patients with migraine without aura and controls without migraine (10). Previous work of Aurora and Mulleners showed that healthy controls exhibited the largest suppression at $100 \mathrm{~ms}$ SOA, followed by migraine patients without aura, while chronic migraine patients and episodic migraineurs with aura showed the least suppression $(12,13)$. Consistently, within the VSS group, subjects with migraine with aura showed a smaller MSPA compared to those without aura. However, the difference was not significant, maybe due to the small sample sizes in the subgroups.

It has been discussed that a reduced MSPA reflects a higher cortical excitability due to a deficiency of intracortical inhibition of the primary visual cortex (12). This would facilitate the initiation of cortical spreading depression, resulting in an attack of migraine with visual aura, but apparently playing a minor role in migraineurs without aura $(10,14)$.

Visual snow syndrome is thought to involve cortical hyperexcitability or a lack of inhibition. The present results suggest that, at least for our collective, such hyperexcitability does not seem to arise from the primary visual cortex. From the clinical description, the typical visual phenomena seem to be best explained by a dysfunction of higher-order visual cortex. 
This is supported by overlapping morphological and functional correlates in the visual association cortex in PET and MRI $(3,6)$ as well as alterations in the late visual evoked potentials (4).

\section{LIMITATIONS}

One limitation of the study is the lack of matching for gender, but to the best of our knowledge, there is no evidence of sex differences in MSPA. Nevertheless, an influence cannot be excluded. Another limitation is the lack of matching for aura, but based on our explorative subgroup analysis irrespective of inclusion or exclusion of the aura patients in the VSS group, the results remained unchanged. Additionally, it should be mentioned that, in the VSS group, the disability caused by headache measured by Migraine Disability Assessment was lower compared to the migraine-matched controls, as we matched for comorbidity and not severity. Lastly, we could have added a healthy control group without comorbid migraine and give more details on clinical data.

\section{CONCLUSION}

This study demonstrates that magnetic suppression of perceptual accuracy, in contrast to the situation in migraine with aura, is not reduced in VSS compared to migraine-matched controls. Therefore, although hyperexcitability apparently occurs in both VSS and migraine aura, the locations seem to be different. The primary visual cortex might not be the main location in VSS.

\section{DATA AVAILABILITY STATEMENT}

The raw data supporting the conclusions of this article will be made available by the authors, without undue reservation.

\section{REFERENCES}

1. Bessero AC, Plant GT. Should "visual snow" and persistence of after-images be recognised as a new visual syndrome? J Neurol Neurosurg Psychiatry. (2014) 85:1057-8. doi: 10.1136/jnnp-2013-306827

2. Schankin C, Maniyar FH, Digre KB, Goadsby PJ. "Visual snow" - a disorder distinct from persistent migraine aura. Brain. (2014) 137:141928. doi: 10.1093/brain/awu050

3. Schankin C, Maniyar F, Sprenger T, Chou DE, Eller M, Goadsby PJ. The relation between migraine, typical migraine aura and visual snow (TM). Headache. (2014) 54:8. doi: 10.1111/head. 12378

4. Eren O, Rauschel V, Ruscheweyh R, Straube A, Schankin CJ. Evidence of dysfunction in the visual association cortex in visual snow syndrome. Ann Neurol. (2018) 84:946-9. doi: 10.1002/ana.25372

5. Puledda F, Schankin C, Digre K, Goadsby P. Visual snow syndrome: what we know so far. Curr Opin Neurol. (2018) 31:52-8. doi: 10.1097/WCO.0000000000000523

6. Schankin CJ, Maniyar FH, Chou DE, Eller M, Sprenger T, Goadsby PJ. Structural and functional footprint of visual snow syndrome. Brain. (2020) 143:1106-13. doi: 10.1093/brain/awaa053

7. Aldusary N, Traber GL, Freund P, Fierz FC, Weber KP, Baeshen A, et al. Abnormal connectivity and brain structure in patients with visual snow. Front Hum Neurosci. (2020) 14:31. doi: 10.3389/fnhum.2020.5 82031

\section{ETHICS STATEMENT}

The studies involving human participants were reviewed and approved by Ludwig Maximilians University Munich (22715). The patients/participants provided their written informed consent to participate in this study.

\section{AUTHOR CONTRIBUTIONS}

AS, CS, OE, RR, TE, and VR contributed to the conception and design of the study. CS, OE, RR, and VR contributed to the acquisition and analysis of data. AS, CS, and OE contributed to the drafting of a significant portion of the manuscript. All authors contributed to the article and approved the submitted version.

\section{FUNDING}

This study received funding from Deutsche Migräneund Kopfschmerzgesellschaft (www.dmkg.de), Eye on Vision Foundation (www.eyeonvision.org), Baasch Medicus Foundation, and Friedrich-Baur Foundation. This study was supported by the self-help group for visual snow (Eye On Vision Foundation) by communicating the study to the patients.

\section{ACKNOWLEDGMENTS}

We thank all the patients who have taken part in the study.

\section{SUPPLEMENTARY MATERIAL}

The Supplementary Material for this article can be found online at: https://www.frontiersin.org/articles/10.3389/fneur. 2021.658857/full\#supplementary-material

8. Lauschke JL, Plant GT, Fraser CL. Visual snow: a thalamocortical dysrhythmia of the visual pathway? J Clin Neurosci. (2016) 28:1237. doi: 10.1016/j.jocn.2015.12.001

9. Unal-Cevik I, Yildiz FG. Visual snow in migraine with aura: further characterization by brain imaging, electrophysiology, and treatment - case report. Headache. (2015) 55:1436-41. doi: 10.1111/head.12628

10. Rauschel V, Ruscheweyh R, Eggert T, Straube A. Magnetic suppression of perceptual accuracy is not reduced in episodic migraine without aura. $J$ Headache Pain. (2014) 15:83. doi: 10.1186/1129-2377-15-83

11. Eren O, Rauschel V, Ruscheweyh R, Straube A, Schankin C. Visual snow syndrome is associated with reduced amplitudes and lack of habituation of visual evoked potentials independent from comorbid migraine. Cephalalgia. (2017) 225-6. doi: 10.1177/0333102417719590

12. Mulleners WM, Chronicle EP, Palmer JE, Koehler PJ, Vredeveld JW. Suppression of perception in migraine: evidence for reduced inhibition in the visual cortex. Neurology. (2001) 56:178-83. doi: 10.1212/WNL.56.2.178

13. Aurora SK, Barrodale P, Chronicle EP, Mulleners WM. Cortical inhibition is reduced in chronic and episodic migraine and demonstrates a spectrum of illness. Headache. (2005) 45:546-52. doi: 10.1111/j.1526-4610.2005.05108.x

14. Charles AC, Baca SM. Cortical spreading depression and migraine. Nat Rev Neurol. (2013) 9:637-44. doi: 10.1038/nrneurol. 2013.192

Conflict of Interest: OE reports grants from Friedrich-Baur Foundation, Deutsche Migräne- und Kopfschmerzgesellschaft during the conduct of the study and 
Novartis outside the submitted work. RR reports personal fees and/or other from Allergan, Novartis, Teva Pharmaceuticals, Hormosan outside the submitted work. CS reports grants from Deutsche Migräne- und Kopfschmerzgesellschaft, Eye on Vision Foundation, and Baasch Medicus Foundation during the conduct of the study, personal fees from Novartis, Eli Lilly, Allergan, Almirall, Amgen, MindMed, and Grünenthal, and personal fees and other from Teva Pharmaceuticals outside the submitted work. AS reports personal fees from Allergan, Bayer, Sanofi, Desitin, Electrocore, Eli Lilly, and Teva Pharmaceuticals and grants from the German Research Council, Kröner-Fresenius Foundation, Ludwig-Maximilian University, and Friedrich-Baur Foundation outside the submitted work.
The remaining authors declare that the research was conducted in the absence of any commercial or financial relationships that could be construed as a potential conflict of interest.

Copyright $\odot 2021$ Eren, Ruscheweyh, Rauschel, Eggert, Schankin and Straube. This is an open-access article distributed under the terms of the Creative Commons Attribution License (CC BY). The use, distribution or reproduction in other forums is permitted, provided the original author(s) and the copyright owner(s) are credited and that the original publication in this journal is cited, in accordance with accepted academic practice. No use, distribution or reproduction is permitted which does not comply with these terms. 